\title{
Basic integrated modelling: a case study
}

\author{
Mario E. Salgado and Diego R. Oyarzún \\ Department of Electronic Engineering, Universidad Técnica Federico Santa María, \\ Valparaíso, Chile \\ E-mail:msb@elo.utfsm.cl
}

\begin{abstract}
A control lab experiment is presented where the key issue is the modelling of a coupled tank system using a suitable combination of phenomenological knowledge and basic least-squares techniques. This approach can be followed without major difficulties by undergraduate students having no prior knowledge of modelling techniques. The paper includes an outline for the lab experiment.
\end{abstract}

Keywords control experiments; least-squares; modelling

One of the key issues in control system design is the availability of a good plant model. This model is generally the starting point in the iterative procedure to build a suitable controller. Most of the time, the required model is a linear model around a chosen operating point. However, when attending a first course on automatic control, the students have no prior knowledge of advanced modelling methods such as least-squares algorithms, ${ }^{1,2}$ since those techniques are not often part of an undergraduate curriculum. The least-squares algorithm is the most popular technique for building models using the input-output or black-box approach. An alternative modelling approach is based on first principles; ${ }^{3}$ this approach provides an insight into the structure and dynamic complexity of a good model, although it demands significant phenomenological knowledge and accurate measurements. Also, a good deal of computing is required to obtain numerical values for the model parameters.

One can imagine the black-box approach and the full phenomenological modelling as the two extremes of a line which includes different degrees of mixing.

In this paper we develop a case study to illustrate how first-principles and basic least-squares techniques can be combined to build a model which is suitable for control and also to study the system behaviour. This can be done for engineering students attending a first lab course on automatic control, having a basic background in signals and systems. The plant we consider is a standard coupled-tank device. This type of system has been widely used in automatic control teaching and for different purposes, due to its simplicity, suitable timescale and reliable actuator and sensors. $^{4-9}$

\section{Plant description and phenomenological modelling}

The plant shown in Fig. 1 is built with two interconnected tanks filled with water, having two positive displacement pumps: a feeding pump P1 and a draining pump P2. Each tank has a cross-sectional surface of $100 \mathrm{~cm}^{2}$ and the pumps are electrically driven and can be used to control the water levels in the tanks. The water levels are measured with semiconductor sensors S1 and S2. The system also has a 


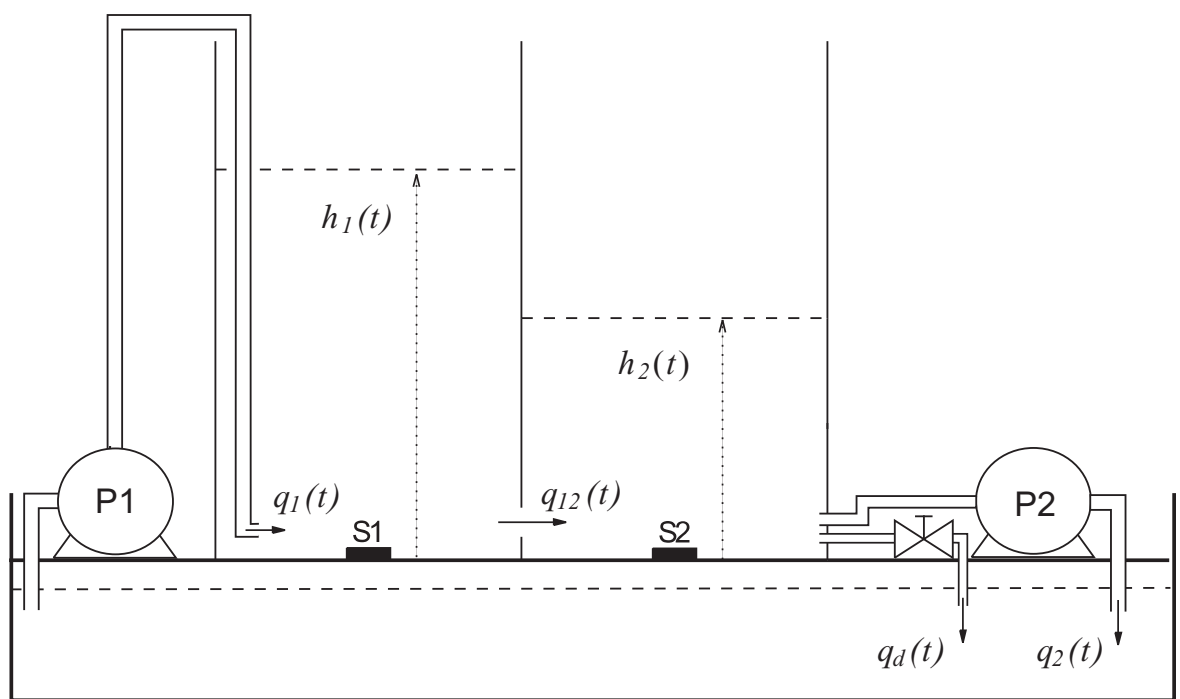

Fig. 1 Coupled tanks system.

manually operated discharge valve which regulates the flow $q_{d}(t)$. The valve is modelled by a parameter $K_{d} \in\left[0 ; K_{\max }\right]$, which depends on the opening of the valve; in the sequel we will assume that $K_{d}$ is either 0 or a fixed non-zero value, to be estimated.

\section{Model}

Tables 1 and 2 below define the variables and the constants required to model the system.

To build a first-principles based model we apply the mass conservation principle. It can then be shown that

$$
\begin{aligned}
& \frac{d m_{1}(t)}{d t}=\rho q_{1}(t)-\rho q_{12}(t) \\
& \frac{d m_{2}(t)}{d t}=\rho q_{12}(t)-\rho q_{2}(t)-\rho q_{d}(t)
\end{aligned}
$$

Where $m_{1}(t), m_{2}(t)$ denote the mass of water in each tank respectively. If the mass is expressed in terms of geometry and density it follows that

$$
\begin{aligned}
& \rho A \frac{d h_{1}(t)}{d t}=\rho q_{1}(t)-\rho q_{12}(t) \\
& \rho A \frac{d h_{2}(t)}{d t}=\rho q_{12}(t)-\rho q_{2}(t)-\rho q_{d}(t)
\end{aligned}
$$


TABLE 1 Dynamical variables involved in system of Fig. 1

\begin{tabular}{lll}
\hline Name & Description & Unit \\
\hline$h_{1}(t)$ & Water level in tank 1 & $\mathrm{cm}$ \\
$h_{2}(t)$ & Water level in tank 2 & $\mathrm{cm}$ \\
$q_{1}(t)$ & Input water flow in tank 1 & $\mathrm{cm}^{3} \mathrm{~s}^{-1}$ \\
$q_{2}(t)$ & Output water flow in tank 2 & $\mathrm{cm}^{3} \mathrm{~s}^{-1}$ \\
$q_{12}(t)$ & Intermediate water flow & $\mathrm{cm}^{3} \mathrm{~s}^{-1}$ \\
$q_{d}(t)$ & Discharge water flow & $\mathrm{cm}^{3} \mathrm{~s}^{-1}$ \\
$v_{h 1}(t)$ & Output voltage in sensor 1 & $\mathrm{V}$ \\
$v_{h 2}(t)$ & Output voltage in sensor 2 & $\mathrm{V}$ \\
$u_{p 1}(t)$ & Voltage applied to pump 1 & $\mathrm{V}$ \\
$u_{p 2}(t)$ & Voltage applied to pump 2 & $\mathrm{V}$ \\
\hline
\end{tabular}

TABLE 2 Constants involved in system of Fig. 1

\begin{tabular}{lll}
\hline Name & Description & Unit \\
\hline$\rho$ & Water density & $\mathrm{g} \mathrm{cm}^{-3}$ \\
$A$ & Cross-sectional surface of each tank & $\mathrm{cm}^{2}$ \\
$g$ & Acceleration of gravity & $\mathrm{cm} \mathrm{s}^{-2}$ \\
$K_{12}$ & Intermediate restriction coefficient & $\mathrm{cm}^{5 / 2} \mathrm{~s}^{-1}$ \\
$K_{d}$ & Discharge restriction coefficient & $\mathrm{cm}^{5 / 2} \mathrm{~s}^{-1}$ \\
$K_{m 1}$ & Gain of level sensor 1 & $\mathrm{V} \mathrm{cm}$ \\
$K_{m 2}$ & Gain of level sensor 2 & $\mathrm{V} \mathrm{cm}$ \\
$\delta_{1}$ & Offset in level sensor 1 & $\mathrm{V}$ \\
$\delta_{2}$ & Offset in level sensor 2 & $\mathrm{V}$ \\
$K_{p 1}$ & Gain of pump 1 power amplifier & $\mathrm{cm}^{3} \mathrm{~V}^{-1} \mathrm{~s}^{-1}$ \\
$K_{p 2}$ & Gain of pump 2 power amplifier & $\mathrm{cm}^{3} \mathrm{~V}^{-1} \mathrm{~s}^{-1}$ \\
$\delta_{p 1}$ & Offset of pump 1 power amplifier & $\mathrm{cm}^{3} \mathrm{~s}^{-1}$ \\
$\delta_{p 2}$ & Offset of pump 2 power amplifier & $\mathrm{cm}^{3} \mathrm{~s}^{-1}$ \\
\hline
\end{tabular}

Using basic fluid mechanics principles (Bernoulli's law in this case ${ }^{3}$ ) plus some geometric simplification, it can be proved that the influence of the orifice connecting both tanks can be modelled as a nonlinear fluid resistor. The same principles apply to the phenomenological modelling of the discharge. We thus have

$$
\begin{aligned}
& q_{12}(t)=K_{12} \sqrt{h_{1}(t)-h_{2}(t)} \\
& q_{d}(t)=K_{d} \sqrt{h_{2}(t)}
\end{aligned}
$$

Combining the above equations, a model is obtained as:

$$
\begin{aligned}
& A \frac{d h_{1}(t)}{d t}=-K_{12} \sqrt{h_{1}(t)-h_{2}(t)}+q_{1}(t) \\
& A \frac{d h_{2}(t)}{d t}=K_{12} \sqrt{h_{1}(t)-h_{2}(t)}-K_{d} \sqrt{h_{2}(t)}-q_{2}(t)
\end{aligned}
$$


Two issues remain to be addressed: we need to link the liquid heights $h_{1}(t)$ and $h_{2}(t)$ with the outputs of the level sensors, and the pump flows $q_{1}(t)$ and $q_{2}(t)$ with the corresponding voltage applied to each pump. These instrumentation relationships, provided by the manufacturers, are

$$
\begin{array}{ll}
v_{h 1}(t)=K_{m 1} h_{1}(t)+\delta_{1} & v_{h 2}(t)=K_{m 2} h_{2}(t)+\delta_{2} \\
q_{1}(t)=K_{p 1} u_{p 1}(t)+\delta_{p 1} & q_{2}(t)=K_{p 2} u_{p 2}(t)+\delta_{p 2}
\end{array}
$$

Note that the description for the draining pump, that is the equation for $q_{2}(t)$, does not consider the effect of $h_{2}(t)$ in the pump flow.

To design a linear controller, such as a member of the PID family, the standard approach is to build an incremental (linearised) model around an equilibrium point. Assume that this equilibrium point $Q$ is achieved with a pair of constant pump voltages $U_{p 1 Q}$ and $U_{p 2 Q}$. Then, the remaining equilibrium values, assuming that the water always flows from tank 1 to tank 2 , are given by

$$
\begin{aligned}
& Q_{1 Q}=K_{p 1} U_{p 1 Q}+\delta_{p 1} \quad Q_{2 Q}=K_{p 2} U_{p 2 Q}+\delta_{p 2} \quad Q_{d Q}=Q_{1 Q}-Q_{2 Q} \\
& H_{2 Q}=\frac{Q_{d Q}^{2}}{K_{d}^{2}} \quad H_{1 Q}=\frac{Q_{1 Q}^{2}}{K_{12}^{2}}+H_{2 Q} \quad Q_{12 Q}=K_{12} \sqrt{H_{1 Q}-H_{2 Q}}
\end{aligned}
$$

If we next use incremental variables, having the generic form $\Delta f(t)=f(t)-F_{Q}$, the linearised model built from (7)-(8) becomes

$$
\begin{aligned}
& A \frac{d \Delta h_{1}(t)}{d t}=-\frac{K_{12}}{2 \sqrt{H_{1 Q}-H_{2 Q}}}\left(\Delta h_{1}(t)-\Delta h_{2}(t)\right)+\Delta q_{1}(t) \\
& A \frac{d \Delta h_{2}(t)}{d t}=\frac{K_{12}}{2 \sqrt{H_{1 Q}-H_{2 Q}}}\left(\Delta h_{1}(t)-\Delta h_{2}(t)\right)-\frac{K_{d}}{2 \sqrt{H_{2 Q}}} \Delta h_{2}(t)-\Delta q_{2}(t)
\end{aligned}
$$

And, on introducing the sensor and pump equations (9) and (10), the overall linearised model becomes

$$
\begin{aligned}
\frac{A}{K_{m 1}} \frac{d \Delta v_{h 1}(t)}{d t}= & -\frac{K_{12}}{2 \sqrt{H_{1 Q}-H_{2 Q}}}\left(\frac{\Delta v_{h 1}(t)}{K_{m 1}}-\frac{\Delta v_{h 2}(t)}{K_{m 2}}\right)+K_{p 1} \Delta u_{p 1}(t) \\
\frac{A}{K_{m 2}} \frac{d \Delta v_{h 2}(t)}{d t}= & \frac{K_{12}}{2 \sqrt{H_{1 Q}-H_{2 Q}}}\left(\frac{\Delta v_{h 1}(t)}{K_{m 1}}-\frac{\Delta v_{h 2}(t)}{K_{m 2}}\right) \\
& -\frac{K_{d}}{2 K_{m 2} \sqrt{H_{2 Q}}} \Delta v_{h 2}(t)-K_{p 2} \Delta u_{p 2}(t)
\end{aligned}
$$

Equations (15) and (16) describe a linear 2-input, 2-output model, where the inputs are the incremental pump voltages $\Delta u_{p 1}(t)$ and $\Delta u_{p 2}(t)$, and the outputs are the incremental level sensor outputs, $\Delta v_{h 1}(t)$ and $\Delta v_{h 2}(t)$. Alternatively, the model above can also be interpreted as a state description of the system, where the states are $\Delta v_{h 1}(t)$ and $\Delta v_{h 2}(t)$.

Regardless of the interpretation we give to this description, to obtain a model which is useful for control design, numerical values for the system parameters are 
first needed. At first glance, these values could be computed from the operatingpoint equations, provided that the equilibrium values could be measured. However, two issues must be considered, namely:

- Every measurement is noisy, hence the computation of the parameters based on a single measurement of every variable can have significant errors.

- The model above has limited fidelity, hence an optimisation approach is highly advisable to compute the values for the model parameters.

A common strategy is to collect a large set of measurements and use them to compute the parameters so that the resulting model is such that the data is reproduced with minimum error. This is the underlying concept in methods like least-squares estimation (LSE).

In the next section, we briefly present the LSE method, in its basic form, to subsequently apply that method to the numerical construction of the system model.

\section{Least squares}

Assume that we have a collection of data and that, based on phenomenological reasoning, we conclude that a good model is

$$
y[k]=\boldsymbol{\varphi}[k]^{T} \boldsymbol{\theta}+\varepsilon[k] \quad k=1,2, \ldots, N
$$

where $y[k] \in \mathbb{R}$ and $\varphi[k] \in \mathbb{R}^{p}$ are functions of data taken from a real process, $\varepsilon[k]$ is an error term, and $\boldsymbol{\theta} \in \mathbb{R}^{p}$ is a vector to be determined so that a measure (cost function) of the error term is minimised. We choose this measure as the quadratic error between the process data and (17), so that the optimal parameter vector $\boldsymbol{\theta}^{\mathbf{0}}$ is:

$$
\boldsymbol{\theta}^{\mathbf{o}}=\left(\boldsymbol{\Phi}^{T} \boldsymbol{\Phi}\right)^{-1} \boldsymbol{\Phi}^{T} \mathbf{Y}_{\mathbf{N}}
$$

where

$$
\begin{aligned}
& \mathbf{Y}_{\mathbf{N}}=[y[1] \quad y[2] \quad \cdots \quad y[N]] \\
& \boldsymbol{\Phi}^{T}=\left[\begin{array}{llll}
\boldsymbol{\varphi}[1] & \boldsymbol{\varphi}[2] & \cdots & \boldsymbol{\varphi}[N]
\end{array}\right]
\end{aligned}
$$

The error term, $\varepsilon[k]$, in equation (17) encompasses measurement errors as well as modelling errors arising from the high complexity of the process description. Here is where the strength of the LSE technique lies, since it implicitly averages, in an optimal way, the modelling errors. This feature connects LSE methods with the optimal filtering theory.

In the current case study we will only deal with, at most, a $2 \times 2$ inverse in (18). This simplicity, plus the fact that we design suitable data collection experiments, makes unnecessary to address in this paper other relevant issues such as invertibility of $\boldsymbol{\Phi}^{T} \boldsymbol{\Phi}$. $^{1}$

A useful feature of the LSE approach is that we only require the model (17) to be linear in the parameters $\boldsymbol{\theta}$, but it can be a non-linear function of the measurements, since $\varphi[k]$ may be a very complex function of the data. This feature will be explored in this case study. 


\section{Parameter estimation}

In this section, values for the model parameters are estimated using the least squares estimation approach. This computation requires measurements of the system variables.

\section{Sensor modelling}

We first estimate the parameters of both sensors modelled in equation (9). This can be done by applying LSE independently to each of the sensors. The key idea is to measure, at regular level intervals, the liquid level $h_{i}$ and the sensor output $v_{h i}$ in tank $i$, for $i=1,2$. If we denote those measurements as $h_{i m}$ and $v_{\text {him }}$, respectively, then

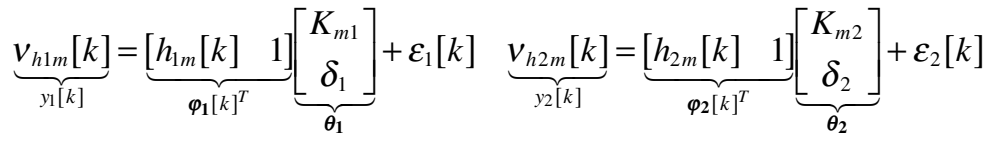

so that the parameters $K_{m 1}, \delta_{1}, K_{m 2}$ and $\delta_{2}$ can be estimated using the LSE method.

In the experiment to obtain the data, a voltmeter is used to measure the each sensor output and a ruler is used to measure the liquid levels. To obtain the data pairs $\left(h_{i m}[k]\right.$, $v_{\text {him }}[k]$ ), both tanks are filled with water (approximately $27 \mathrm{~cm}$ ). Then, with no voltage applied to the pumps, and using the hand-operated discharge, the levels in both tanks are taken down in steps of approximately $2 \mathrm{~cm}$ each time. Recording the voltages and the level in both tanks, the relationships shown in Fig. 2 are obtained. Note that in every step, the measurements must be taken once the levels have settled to the new value. Building the appropriate matrices $\boldsymbol{\Phi}$ and $\mathbf{Y}_{\mathbf{N}}$ for each sensor and using equation (18), the optimal vectors of parameters result:

$$
\boldsymbol{\theta}_{1}=\left[\begin{array}{l}
0.189 \\
0.715
\end{array}\right] \quad \boldsymbol{\theta}_{2}=\left[\begin{array}{l}
0.187 \\
0.597
\end{array}\right]
$$

Then, the sensors input/output relationship can then be modelled as

$$
\begin{array}{ll}
v_{h 1}(t)=\left(0.189 h_{1}(t)+0.715\right)[V] & h_{1}(t) \geq 0[\mathrm{~cm}] \\
v_{h 2}(t)=\left(0.187 h_{2}(t)+0.597\right)[V] & h_{2}(t) \geq 0[\mathrm{~cm}]
\end{array}
$$

\section{Pump modelling}

The parameters in the input/output relationships of both pumps in equation (10) can also be computed by independent application of the LSE methodology. This requires the measurement, at regular voltage intervals, of the pump flows $q_{i}(t)$ and the voltage applied to the pumps, $u_{p i}(t), i=1,2$. If we assume that the measurements are $q_{\mathrm{im}}(t)$ and $u_{\text {pim }}(t)$ respectively, then

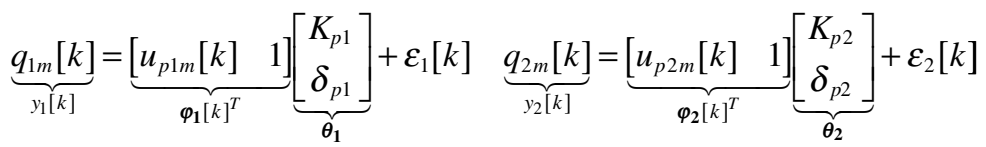




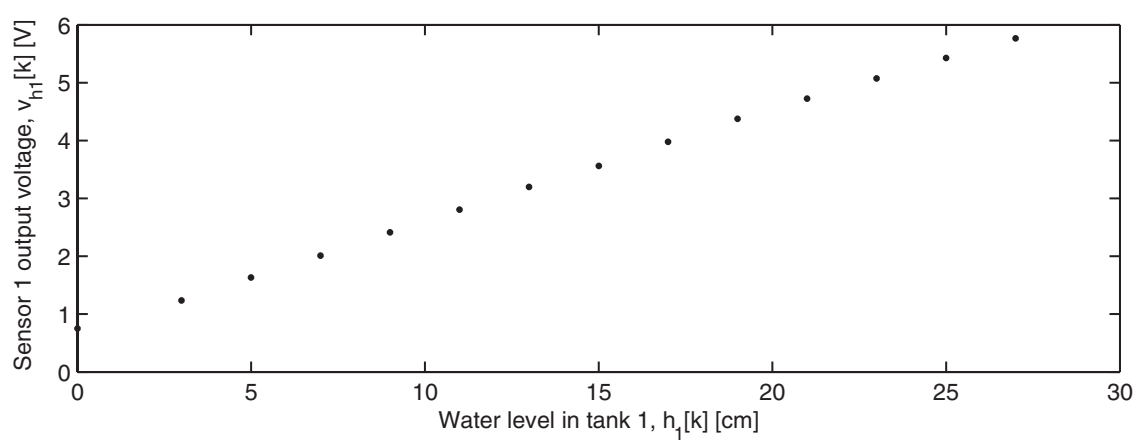

(a)

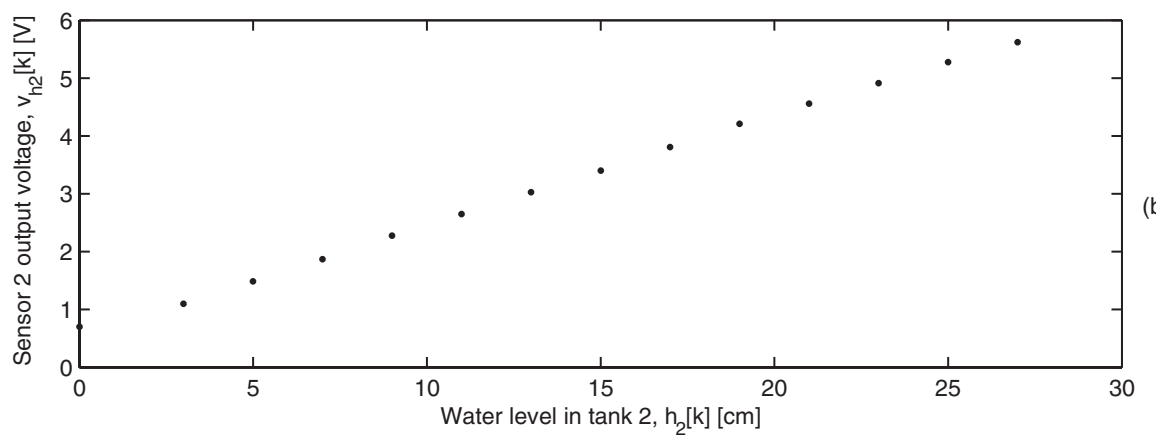

Fig. 2 Voltage-height characteristic of: (a) sensor in tank 1, (b)sensor in tank 2.

so that the parameters $K_{p 1}, K_{p 2}, \delta_{p 1}$ and $\delta_{p 2}$ can be estimated using LSE. However this will require a special sensor to measure flows $q_{1}(t)$ and $q_{2}(t)$. This is hard since in this case, small flows are involved and accurate measurement is then expensive. A way to circumvent this difficulty is to substitute the flow measurement by its firstorder approximation. To build that approximation, we assume that the handoperated valve is closed and that only one pump is working, at a fixed voltage, during a time interval $T$. We measure the total volume of water in both tanks, i.e. the liquid level in both tanks, at the beginning of the interval, and then at the end of the interval, thus

$$
q_{i m}[k] \approx \frac{\operatorname{vol}_{m}[k]-\operatorname{vol}_{m}[k-1]}{T}=\tilde{q}_{i}[k]
$$

the experiment is performed for a voltage set which spans the allowable range. Then the experiment is repeated for the other pump. Figure 3 shows the results of the described experiments for $T=7 \mathrm{~s}$. During the measurements it was discovered that both pumps exhibit a dead zone; however only that of the output pump is significant. The results of the estimation process using (18) yields the following pump models. 


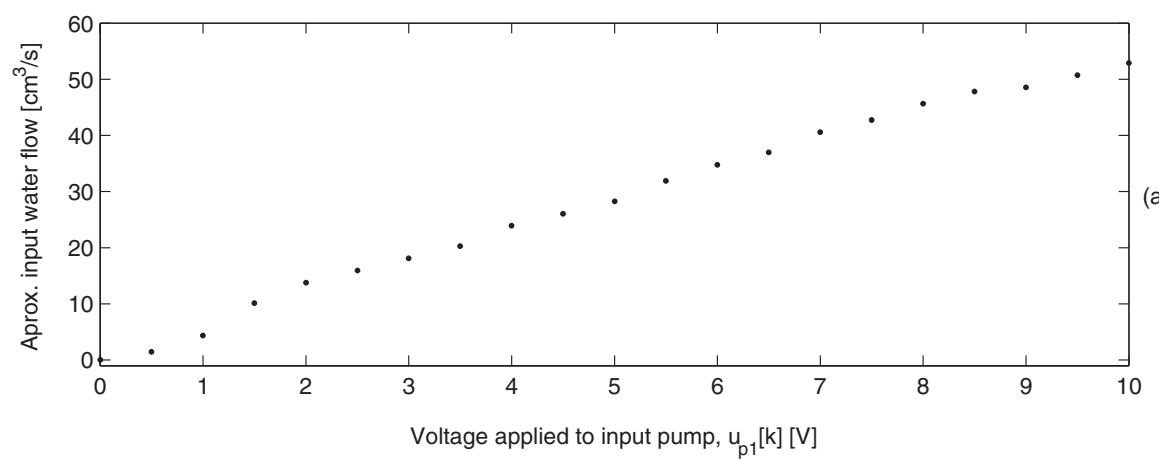

(a)

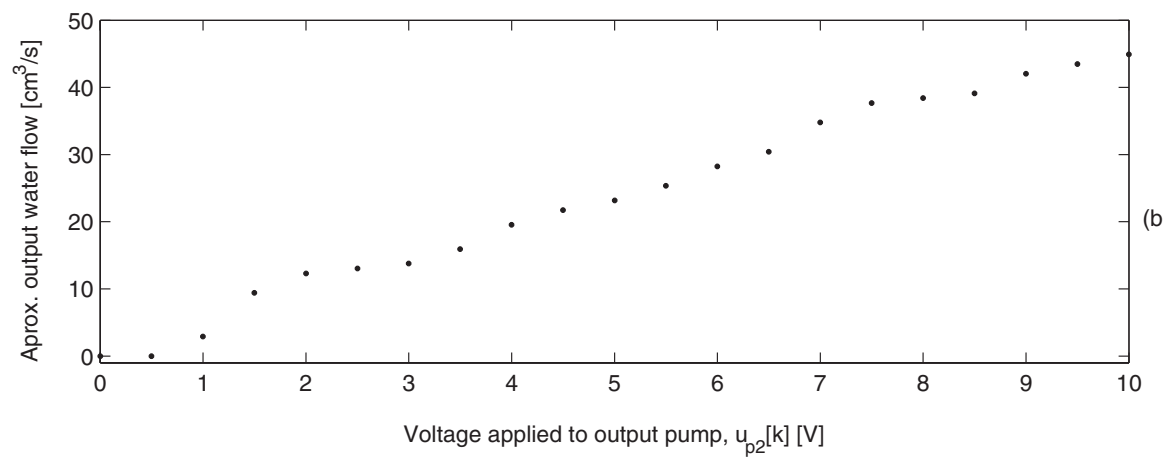

Fig. 3 Flow-voltage characteristic of: (a) input pump, (b) output pump.

$$
\begin{aligned}
& q_{1}(t)= \begin{cases}\left(5.41 u_{p 1}(t)+1.28\right)\left[\mathrm{cm}^{3} / s\right] & u_{p 1}(t)>0[V] \\
0 & u_{p 1}(t) \leq 0[V]\end{cases} \\
& q_{2}(t)= \begin{cases}\left(4.64 u_{p 2}(t)+0.477\right)\left[\mathrm{cm}^{3} / s\right] & u_{p 2}(t)>0.5[V] \\
0 & u_{p 2}(t) \leq 0.5[V]\end{cases}
\end{aligned}
$$

\section{Connecting orifice modelling}

The circular orifice connecting both tanks plays a key role in the overall system dynamics. If the tank section is fixed, then the radius of the orifice determines, together with $K_{d}$, the time constants in the linear model (15)-(16). The parameter $K_{12}$ condenses this dependence. Hence, a larger orifice section will lead to a larger $K_{12}$ which, in turn, will result in faster dynamics.

The experiment to collect the data to be used in the parameter estimation should be carefully designed, while at the same time it should be kept simple. In this experiment we only work with initial conditions, i.e. both pumps are turned off. Firstly, the connecting orifice is closed, the discharge is off $\left(K_{d}=0\right)$ and water is poured into the tanks, so that $h_{1}[0] \gg h_{2}[0]$. Next, the orifice is unplugged and measurements of $h_{1}$ and $h_{2}$ are taken every $T_{o}$ seconds. 
To estimate the orifice constant $K_{12}$ we also need to estimate the flow variable $q_{12}$. We thus use the same strategy as the previous section.

$$
q_{12}[k] \approx \frac{\operatorname{vol}_{2}[k]-\operatorname{vol}_{2}[k-1]}{T_{o}}=-\frac{\operatorname{vol}_{1}[k]-v o l_{1}[k-1]}{T_{o}}=\tilde{q}_{12}[k]
$$

If we denote by $h_{1 m}[k]$ and $h_{2 m}[k]$ the measurements of $h_{1}$ and $h_{2}$, then according to (5) the model where LSE will be applied is

$$
\tilde{q}_{12}[k]=\underbrace{\frac{A}{T_{o}}\left(h_{2 m}[k]-h_{2 m}[k-1]\right)}_{y[k]}=\underbrace{\sqrt{h_{1 m}[k]-h_{2 m}[k]}}_{\phi[k]} \underbrace{K_{12}}_{\theta}+\varepsilon[k]
$$

For instance, if we choose $h_{1}[0]=25.5[\mathrm{~cm}], h_{2}[0]=3[\mathrm{~cm}]$ and $T_{o}=0.03[\mathrm{~s}]$, and if we use the sensors characteristics given in (23) and (24), the data plotted in Fig. 4 are obtained. Note that although the measurement is noisy, the noise is not significantly high. The optimal value for $K_{12}$ using (18) turns out to be

$$
K_{12}=12.47\left[\frac{\mathrm{cm}^{\frac{5}{2}}}{\mathrm{~s}}\right]
$$

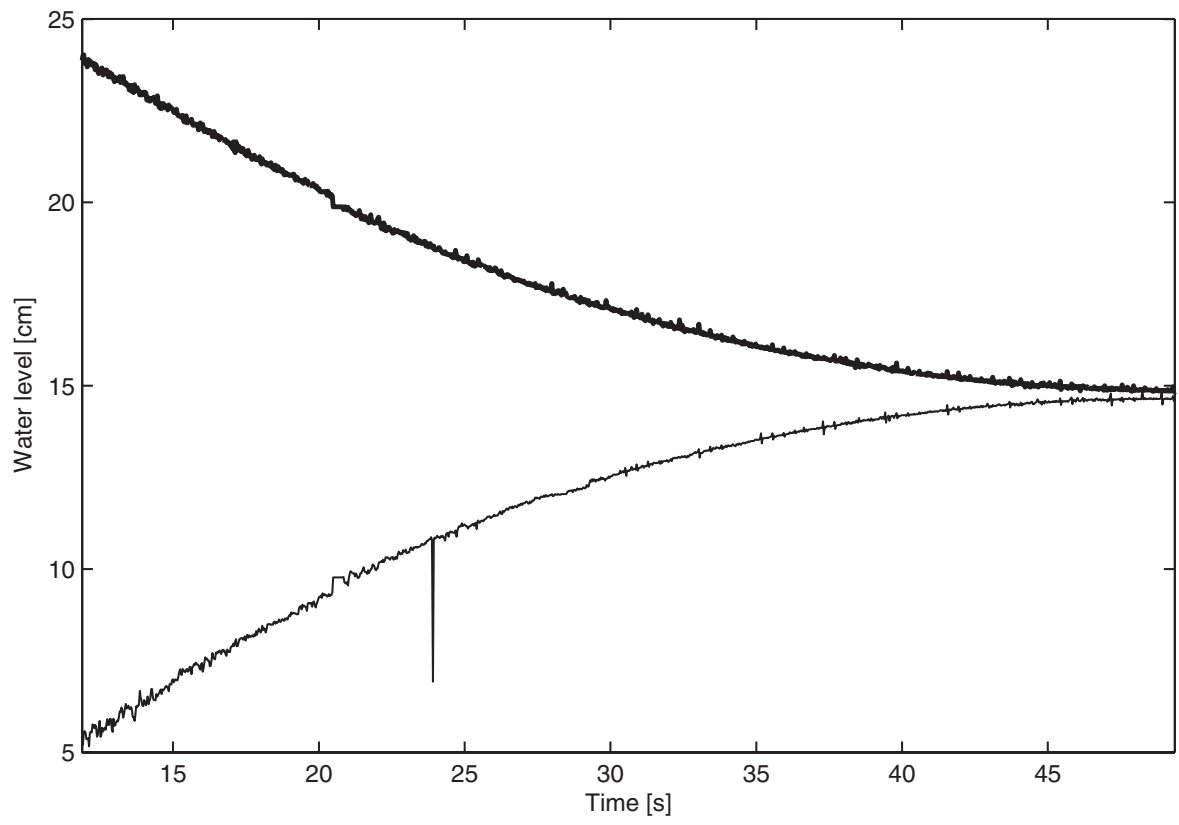

Fig. 4 Measurement of water level in tank 1 (thick) and tank 2 (thin). 


\section{Discharge modelling}

The discharge plays a similar role in the system dynamics to that of the connecting orifice and the strategy to estimate the discharge coefficient $K_{d}$ is the same as the one used to estimate $K_{12}$. For that purpose, both pumps are powered off and the connecting orifice is blocked $\left(K_{12}=0\right)$. Then, water is poured into tank 2 , and the level $h_{2}$ is measured every $T_{d}[s]$ while the tank is being drained. To build a model suitable to apply LSE, we again use a first order approximation for the discharge flow $q_{d}$, namely

$$
q_{d}[k] \approx-\frac{v l_{2}[k]-v o l_{2}[k-1]}{T_{d}}=\tilde{q}_{d}[k]
$$

If we denote by $h_{2 m}[k]$ the measurement of $h_{2}$, then the model where LSE will be applied is

$$
\tilde{q}_{d}[k]=\underbrace{-\frac{A}{T_{d}}\left(h_{2 m}[k]-h_{2 m}[k-1]\right)}_{y[k]}=\underbrace{\sqrt{h_{2 m}[k]}}_{\phi[k]} \underbrace{K_{d}}_{\theta}+\varepsilon[k]
$$

Figure 5 shows the result of an experiment where $h_{2}(t)$ is sampled every $0.03 \mathrm{~s}$. Note that the voltage measurements have been properly converted to level data using (24) and we can see that again the measurement noise is acceptable. The LSE

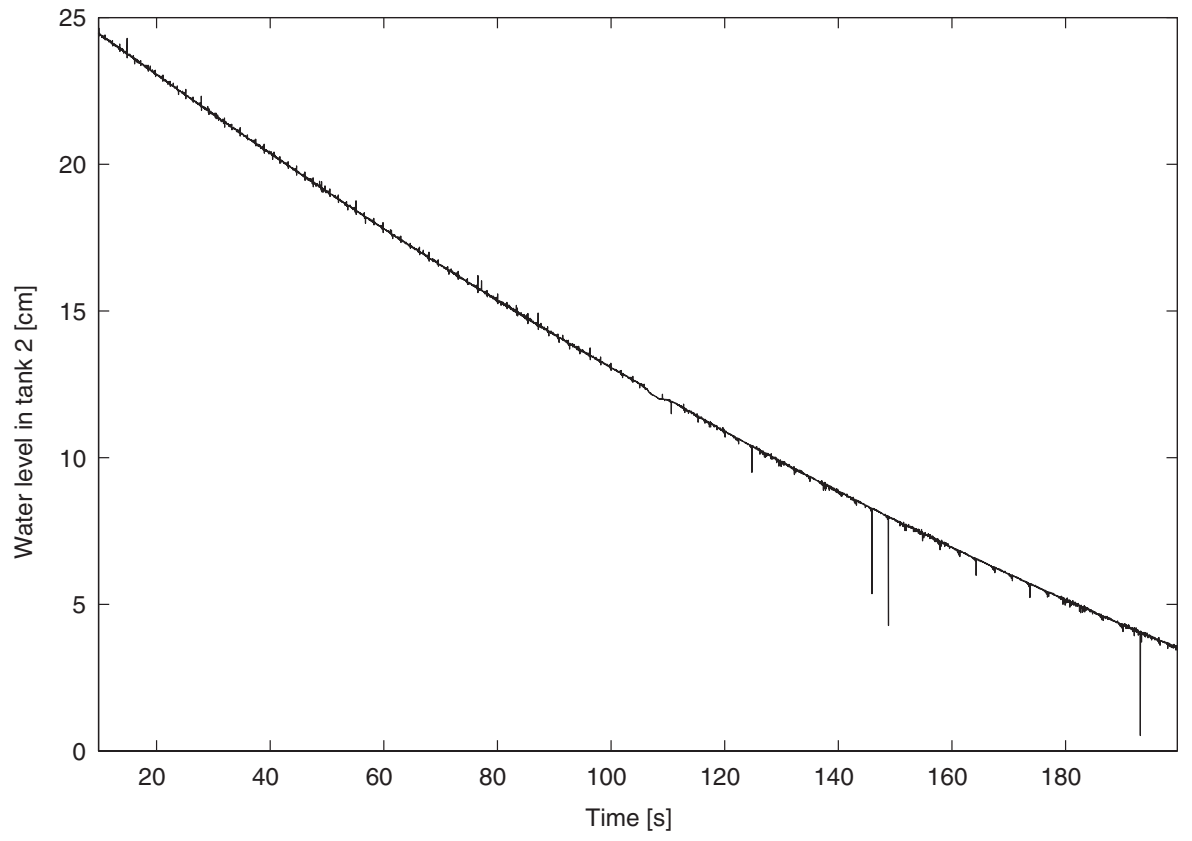

Fig. 5 Measurement of water depleting in tank 2. 
technique is finally applied using the collected data and the optimal value for $K_{d}$ is obtained.

$$
K_{d}=3.497\left[\frac{\mathrm{cm}^{\frac{5}{2}}}{s}\right]
$$

\section{Modelling validation}

Once the model has been completely identified, i.e. all the unknown parameters have been estimated, its effectiveness can be evaluated. The idea of building a mathematical model for a real dynamical system is to achieve the ability to predict, with good accuracy, the response of the real system under different operation conditions, within a sensible range. This means that the usefulness of the model that has been built should be judged for its ability to predict the system response when given inputs are applied. The process of evaluating the prediction performance of the model is known as Model Validation.

The validation is done in two steps. In a first stage, an experiment is performed on the real system. This experiment should be complex enough to reveal all relevant dynamic features in the system response. As a second step, the estimated model is simulated with the same inputs that were applied to the real system, so that by comparing the real and the simulated responses, the accuracy of the model can be assessed.

A validation procedure applied to the coupled tanks system is presented next. Voltages are applied to both pumps and the water levels in both tanks are measured every $0.003 \mathrm{~s}$. Figure 6 shows the input signals applied to each pump and the measured water levels, which have been converted from (23) and (24). Figure 6(b) shows that a significant noise is present in the measured signals. This noise is mainly electromagnetic interference due to the pulse-width modulation used to drive the pumps. To clean the signals for a fair comparison with the model-based simulation, some sort of filtering must be used. One possible approach is to carry out a power spectral density estimate of the measurement noise using some known spectral analysis tools. ${ }^{10}$ However, the theory behind these techniques is far beyond the scope of this paper. Instead we use an ad hoc procedure. To do this we examine Fig. 6(b) in detail. Figure 7 shows the measured level $h_{2}[k]$, zoomed several times. We here observe a very high frequency noise imposed on a lower frequency quasi-periodic signal. The frequency of this quasi-periodic signal can be roughly calculated from the graph, as $0.33 \mathrm{~Hz}$. Therefore a sensible approach is to process the data through a filter which attenuates significantly all frequencies beyond a sensible upper frequency, say $0.3 \mathrm{~Hz}$. A digital third-order low-pass Butterworth filter, ${ }^{11}$ is chosen with a bandwidth of $0.3 \mathrm{~Hz}$. The transfer function of this filter is

$$
H_{F}(z)=\frac{0.0000214 z^{3}+0.0000643 z^{2}+0.0000643 z+0.0000214}{z^{3}-2.887 z^{2}+2.78 z-0.893}
$$

The simulation results of the nonlinear model described by (7)-(10), whose parameter values were estimated in the previous section, are shown in Figs 8(b) and (c) 


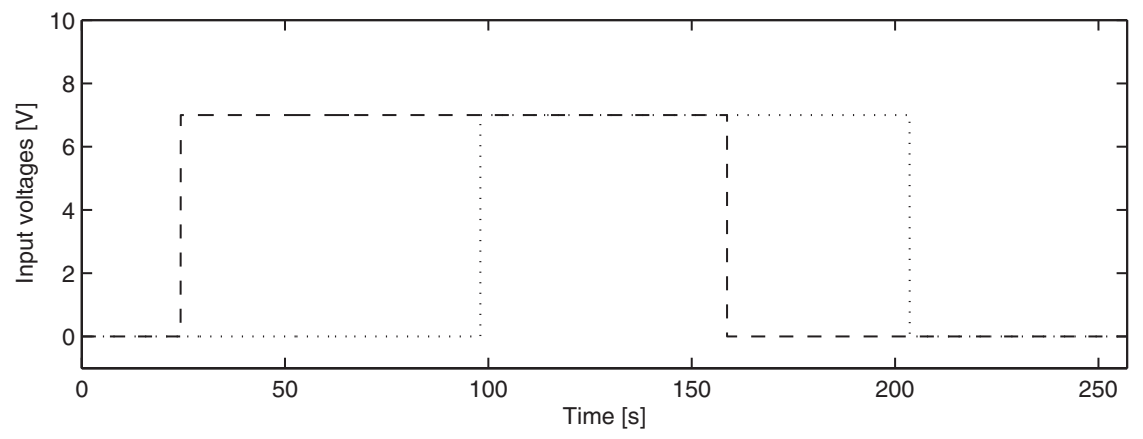

(a)

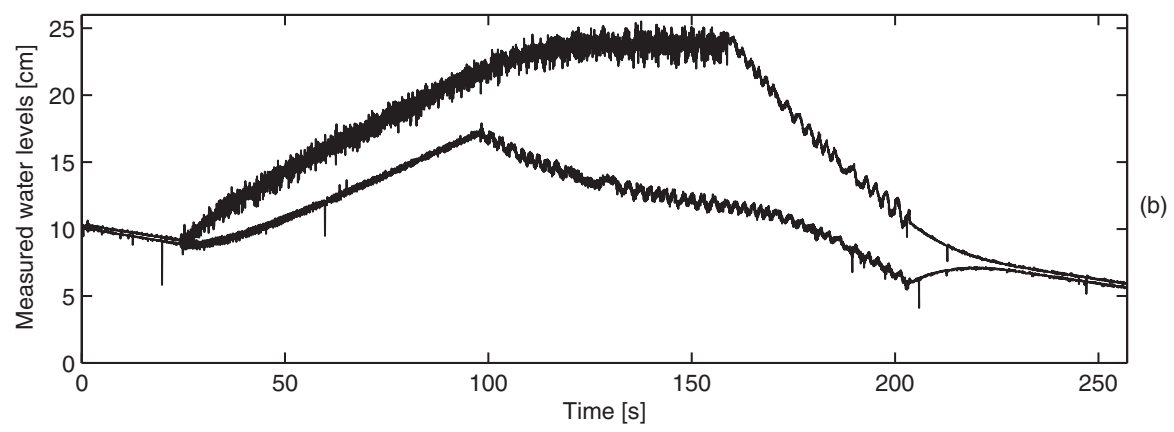

Fig. 6 (a) Input voltage applied to input (dashed) and output (dotted) pumps, (b) measured water level in tank 1 (upper) and tank 2 (lower).

together with the associated filtered measurements. The effect of zero initial conditions in the filter is evident in both signals. It can be seen that the estimated nonlinear model effectively predicts the outputs of the real system.

\section{Prototype lab session}

The material presented in the previous sections can be used in a standard three-hour laboratory session for teaching purposes. The following steps are recommended to exploit in depth the pedagogical features that this case of integrated system modelling provides.

- The students should have completed a previous work, which must accomplish four main objectives:

\section{Knowledge of the hardware involved}

The students must become familiar with the relevant aspects of the coupled tanks plant, actuators, sensors and the digital acquisition system. In the last two items, special attention must be given to the phenomenon of measurement noise. 


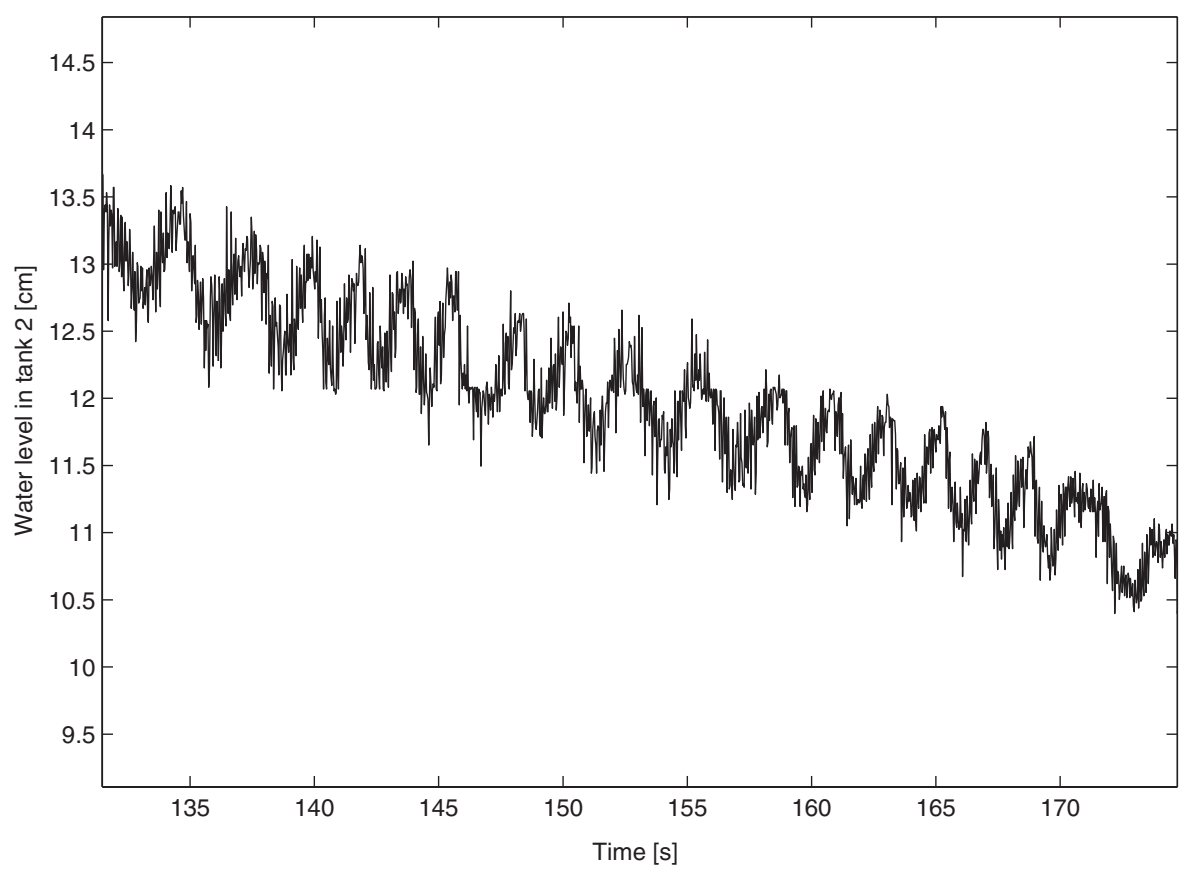

Fig. 7 Zooming of measured tank 2 water level.

Analytical derivation of the nonlinear system model and its linear approximation This corresponds to deriving the model in the subsection 'Model' above.

Knowledge of the basic LSE technique

This can be assisted by a handout covering the material presented in the section 'Least squares'.

Ability to operate the computer software to be used to collect and to process experimental data

The students must propose suitable experiments to collect useful data to perform the estimations and validate the model.

- Within a three-hour lab session, the students should be able to perform the experiments required to estimate all the unknown parameters of the nonlinear model. The experiments should be the ones described in the present paper, but the students could be given bonus marks for trying to compare their own pre-designed experiments.

As well as the experiments needed for the estimation process, the students must perform a validation experiment to assess the quality of the estimated 

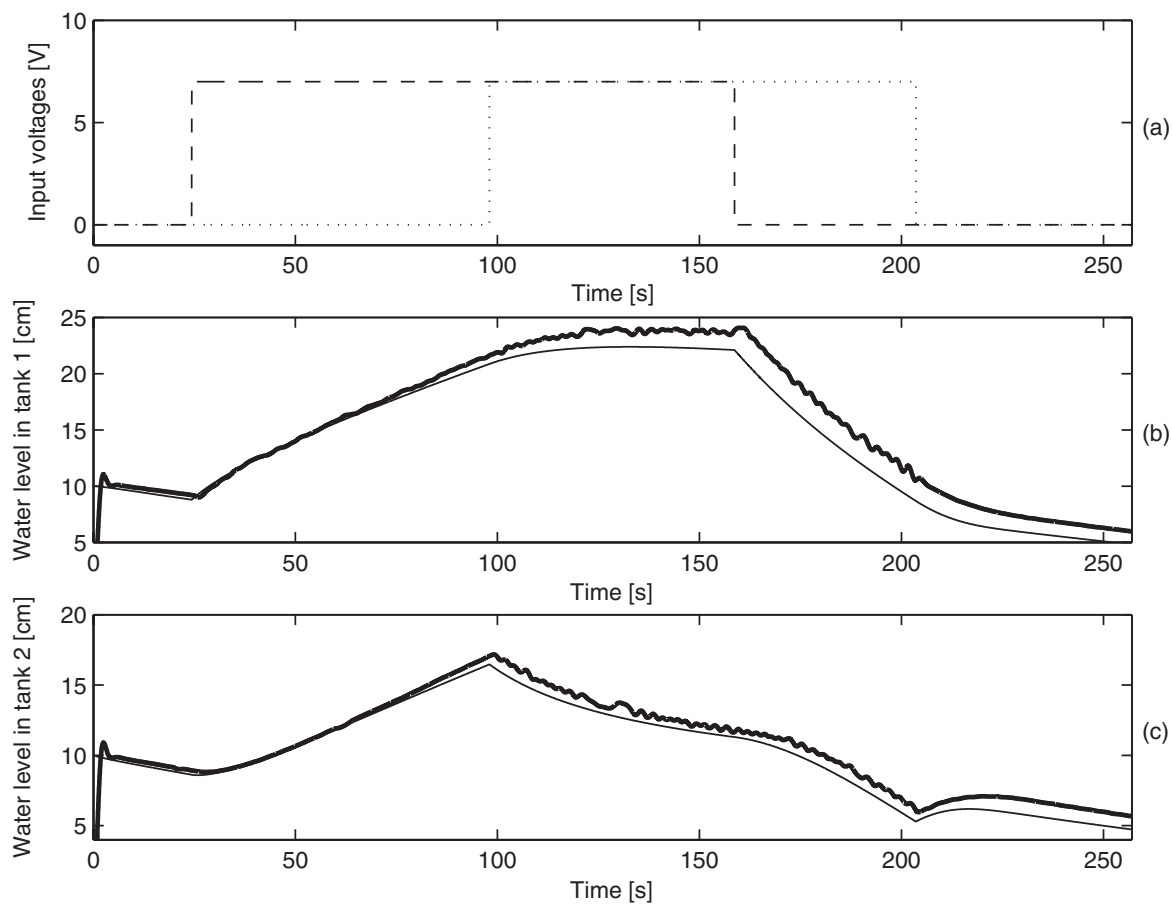

Fig. 8 (a) Input voltage applied to input (dashed) and output (dotted) pumps, (b) filtered measurement of water level in tank 1 (thick) and simulation result (thin), (c) filtered measurement of water level in tank 2 (thick) and simulation result (thin).

TABLE 3 Schematic description of prototype lab session

Task

Est. time

Hardware and software set-up

$20 \mathrm{~min}$

Analysis of pre-designed experiments

$10 \mathrm{~min}$

Experiments to collect data for parameter estimation

$120 \mathrm{~min}$

Experiments to collect data for validation

$30 \mathrm{~min}$

model. The experiment presented in this paper can be taken as a first step, but some variations can be introduced.

The lab session can be organised as shown in Table 3.

- Finally, the estimation and validation procedures must be done at home by students. Note that, as discussed in this paper, some filtering may be needed when the collected data is too noisy. The estimated model must then be simulated with an appropriate software package so that the validation stage can be performed. All these results should be presented in a lab report, together with the relevant conclusions. 


\section{Conclusions}

This paper has presented a case study where phenomenological knowledge has been combined with basic least-squares estimation to obtain a model for a laboratory plant. This approach can be followed by undergraduate students having no previous knowledge of advanced modelling techniques. It has been shown how a set of sensibly designed experiments yield enough data to model sensors, actuators and to estimate the plant's most significant parameters. These estimated parameters can be used to construct a nonlinear description of the system as well as a linearised model around a given equilibrium point. The study has also included a validation stage, to assess the ability of the model to predict the system response under given experimental conditions. In this validation stage, simple techniques have been used to deal with measurement noise. Finally, it is suggested how the content of this paper can be organised in a three-hour lab session with pre- and post-lab work from the students.

\section{Acknowledgements}

The authors gratefully acknowledge support received from UTFSM through a DGIP grant.

\section{References}

1 L. Ljung, System Identification: Theory for the User (Prentice Hall, Upper Saddle River, New Jersey, 1999).

2 R. Pintelon and J. Schoukens, System Identification: A Frequency Domain Approach (Wiley-IEEE Press, New York, 2001).

3 K. Ogata, System Dynamics (Prentice Hall, Englewood Cliffs, New Jersey, 2003).

4 K. J. Åström and A. Östberg, 'A teaching laboratory for process control', IEEE Control Systems Magazine, 6 (1986), 5.

5 A. Baddou, F. Tadeo and A. Benzaouia, 'Two coupled-tanks level regulation with constraints on the control and the output' in E. Camacho, L. Basañez and J. A. de la Puente (Eds), Proc. 15th Triennial IFAC World Congress, Barcelona, 21-26 July, 2002 (Elsevier, Amsterdam).

6 M. Hou, Y. Xiong and R. Patton, 'Observer design for a three-tank system' in E. Camacho, L. Basañez and J. A. de la Puente (Eds), Proc. 15th Triennial IFAC World Congress, Barcelona, 21-26 July, 2002 (Elsevier, Amsterdam).

7 K. H. Johansson, 'The quadruple-tank process: A multivariable laboratory process with an adjustable zero', IEEE Trans. Control Syst. Technol., 8 (2000), 3.

8 R. Pickhardt and C. Schmid, 'Nonlinear adaptive predictive control strategy applied to an interconnected tank system' in E. Camacho, L. Basañez and J. A. de la Puente (Eds), Proc. 15th Triennial IFAC World Congress, Barcelona, 21-26 July, 2002 (Elsevier, Amsterdam).

9 E. Rickebusch and I. Craig, 'PID tuning for a multivariable plant using Taguchi-based methods', in E. Camacho, L. Basañez and J. A. de la Puente (Eds), Proc. 15th Triennial IFAC World Congress, Barcelona, 21-26 July, 2002 (Elsevier, Amsterdam).

10 M. Priestley, Spectral Analysis and Time Series (Academic Press, London, 1981).

11 A. V. Oppenheim, R. Schafer and J. Buck, Discrete-Time Signal Processing (Prentice Hall, Englewood Cliffs, New Jersey, 1999). 